\title{
On the Relaxed Colouring Game and the Unilateral Colouring Game
}

\author{
Elżbieta Sidorowicz
}

Received: 12 March 2012 / Revised: 1 May 2013 / Published online: 31 May 2013

C The Author(s) 2013. This article is published with open access at Springerlink.com

\begin{abstract}
In the paper we introduce the new game - the unilateral $\mathcal{P}$-colouring game which can be used as a tool to study the $r$-colouring game and the $(r, d)$-relaxed colouring game. Let be given a graph $G$, an additive hereditary property $\mathcal{P}$ and a set $C$ of $r$ colours. In the unilateral $\mathcal{P}$-colouring game similarly as in the $r$-colouring game, two players, Alice and Bob, colour the uncoloured vertices of the graph $G$, but in the unilateral $\mathcal{P}$-colouring game Bob is more powerful than Alice. Alice starts the game, the players play alternately, but Bob can miss his move. Bob can colour the vertex with an arbitrary colour from $C$, while Alice must colour the vertex with a colour from $C$ in such a way that she cannot create a monochromatic minimal forbidden subgraph for the property $\mathcal{P}$. If after $|V(G)|$ moves the graph $G$ is coloured, then Alice wins the game, otherwise Bob wins. The $\mathcal{P}$-unilateral game chromatic number, denoted by $\chi_{u g}^{\mathcal{P}}(G)$, is the least number $r$ for which Alice has a winning strategy for the unilateral $\mathcal{P}$-colouring game with $r$ colours on $G$. We prove that the $\mathcal{P}$-unilateral game chromatic number is monotone and is the upper bound for the game chromatic number and the relaxed game chromatic number. We give the winning strategy for Alice to play the unilateral $\mathcal{P}$-colouring game. Moreover, for $k \geq 2$ we define a class of graphs $\mathcal{H}_{k}=\{G \mid$ every block of $G$ has at most $k$ vertices $\}$. The class $\mathcal{H}_{k}$ contains, e.g., forests, Husimi trees, line graphs of forests, cactus graphs. Let $\mathcal{S}_{d}$ be the class of graphs with maximum degree at most $d$. We find the upper bound for the $\mathcal{S}_{2}$-unilateral game chromatic number for graphs from $\mathcal{H}_{3}$ and we study the $\mathcal{S}_{d}$-unilateral game chromatic number for graphs from $\mathcal{H}_{4}$ for $d \in\{2,3\}$. As the conclusion from these results we obtain the result for the $d$-relaxed game chromatic number: if $G \in \mathcal{H}_{k}$, then $\chi_{g}^{(d)}(G) \leq k+2-d$, for $k \in\{3,4\}$ and $d \in\{0, \ldots, k-1\}$. This generalizes a known result for trees.
\end{abstract}

\footnotetext{
E. Sidorowicz $(\varangle)$

University of Zielona Góra, ul. Szafrana 4a, 65-516 Zielona Góra, Poland

e-mail: e.sidorowicz@wmie.uz.zgora.pl
} 
Keywords Colouring game $\cdot$ Relaxed colouring game ·

Generalized colouring game

\section{Introduction}

We consider finite undirected graphs without loops and multiple edges. A graph property is any nonempty proper isomorphism-closed subclass of all graphs. We also say that a graph has the property $\mathcal{P}$ if $G \in \mathcal{P}$. A property $\mathcal{P}$ is called hereditary if it is closed under taking subgraphs, i.e., if $H \subseteq G$ and $G \in \mathcal{P}$, then $H \in \mathcal{P}$. A property $\mathcal{P}$ is called additive if for each graph $G$ all of whose components have the property $\mathcal{P}$ it follows that $G$ has the property $\mathcal{P}$, too. Any hereditary property can be uniquely determined by the set $\mathbf{F}(\mathcal{P})$ of minimal forbidden subgraphs, i.e., $\mathbf{F}(\mathcal{P})=\{G \mid G \notin \mathcal{P}$ but each proper subgraph of $G$ belongs to $\mathcal{P}\}$. If $\mathcal{P}$ is an additive hereditary property, then every graph in $\mathbf{F}(\mathcal{P})$ is connected. In this paper only additive hereditary properties are considered.

Let be given a graph $G$ and an ordered set of additive hereditary properties $\left(\mathcal{P}_{1}\right.$, $\mathcal{P}_{2}, \ldots, \mathcal{P}_{r}$ ). Two players Alice and Bob colour alternately the uncoloured vertices of a graph $G$ from the set of $r$ colours $C$. Alice starts the game. The player may colour a vertex $v$ with colour $i \in\{1, \ldots, r\}$ if after the move a subgraph induced by vertices with colour $i$ has the property $\mathcal{P}_{i}$. If after $|V(G)|$ moves the graph $G$ is coloured, then Alice wins, otherwise Bob wins. The above game called a $\left(\mathcal{P}_{1}, \mathcal{P}_{2}, \ldots, \mathcal{P}_{r}\right)$ colouring game (generalized game) was introduced in [3]. In other words, in the $\left(\mathcal{P}_{1}, \mathcal{P}_{2}, \ldots, \mathcal{P}_{r}\right)$-colouring game the players colour vertices such that they never create any monochromatic minimal forbidden subgraph for the property $\mathcal{P}_{i}$ in colour $i$. If $\mathcal{P}_{1}=\mathcal{P}_{2}=\cdots=\mathcal{P}_{r}=\mathcal{P}$, then the game is called the $\mathcal{P}$-colouring game with $r$ colours for short. A $\mathcal{P}$-game chromatic number $\chi_{g}^{\mathcal{P}}(G)$ of $G$ is the least number $r$ for which Alice has a winning strategy for the $\mathcal{P}$-colouring game with $r$ colours.

If $\mathcal{P}$ is a set of edgeless graphs and players use $r$ colours, then we obtain the most known variant of the $\mathcal{P}$-colouring game-the $r$-colouring game. Thus, in the $r$-colouring game the player may colour the vertex $v$ with colour $i \in C$ if $v$ has no neighbours already coloured with $i$. A game chromatic number $\chi_{g}(G)$ of $G$ is the least number $r$ for which Alice has a winning strategy for the $r$-colouring game. The concept of the game chromatic number was introduced in [2]. Since then, the game chromatic number of various classes of graphs has been studied.

The variant of the $\mathcal{P}$-colouring game such that $\mathcal{P}$ is the set of graphs with maximum degree at most $d$, was introduced by Chou et al. [5] and it is called the $(r, d)$ relaxed colouring game. A $d$-relaxed game chromatic number, denoted by $\chi_{g}^{(d)}(G)$, is the smallest $r$ for which Alice has a winning strategy for the $(r, d)$-relaxed colouring game. For a class of graphs $\mathcal{R}$, let $\chi_{g}^{\mathcal{P}}(\mathcal{R})=\max \left\{\chi_{g}^{\mathcal{P}}(G) \mid G \in \mathcal{R}\right\}, \chi_{g}(\mathcal{R})=$ $\max \left\{\chi_{g}(G) \mid G \in \mathcal{R}\right\}, \chi_{g}^{(d)}(\mathcal{R})=\max \left\{\chi_{g}^{(d)}(G) \mid G \in \mathcal{R}\right\}$.

The relaxed colouring game on the classes of partial $k$-trees, planar, and outerplanar graphs, forests have been well studied. Let $\mathcal{T}_{2}$ be the class of outerplanar graphs and $\mathcal{T}_{1}$ be the class of forests. The results for outerplanar graphs proved in a series of papers $[5,11,12,16]$ can be expressed in the following formula. 
Theorem 1 ([5, 11, 12,16]) For $d \in\{0,1,2,3,4\}$ it holds that $\chi_{g}^{(d)}\left(\mathcal{T}_{2}\right) \leq 7-d$.

Moreover, Wu and Zhu in [17] showed that $\chi_{g}^{(d)}\left(\mathcal{T}_{2}\right)=2$ for all $d \geq 6$. A similar uniform formula for forests was proved in a series of papers $[5,10,12]$.

Theorem 2 ([5,10,12]) For $d \in\{0,1,2\}$ it holds that $\chi_{g}^{(d)}\left(\mathcal{T}_{1}\right) \leq 4-d$.

In this paper we find the similar formula for the special class of graphs $\mathcal{H}_{k}$, which contains, e.g., forests, line graphs of forests, cactus graphs, Husimi trees.

A block $B$ of a graph $G$ is a maximal subgraph of $G$ that does not contain any cut-vertex. For $k \geq 2$, let

$$
\mathcal{H}_{k}=\{G \mid \text { every block of } G \text { has at most } k \text { vertices }\}
$$

Since $\mathcal{H}_{k}$ contains many well known classes of graphs, it is worth studying. For example $\mathcal{H}_{2}$ is the class of forests. If $G \in \mathcal{H}_{k}$ and every block is a complete graph, then $G$ is a Husimi tree. If $G \in \mathcal{H}_{k}$ and every block is a cycle or $K_{2}$, then $G$ is a cactus graph. The class $\mathcal{H}_{k}$ is also interesting, because it contains line graphs of forests. If every block of $G \in \mathcal{H}_{k}$ is a complete graph and every vertex of $G$ is in at most two blocks, then $G$ is the line graph of a forest. Thus, the colouring game on vertices of graphs from $\mathcal{H}_{k}$ corresponds to the edge-colouring game on forests. The edge version of the colouring game and the relaxed colouring game was studied in $[1,4,6,9]$.

Since $\mathcal{H}_{2}$ is the class of forests, we can reformulate Theorem 2 in the following way: for $d \in\{0,1,2\}$ it holds that $\chi_{g}^{(d)}\left(\mathcal{H}_{2}\right) \leq 4-d$. In this paper, we extend the class of graphs for which Theorem 2 holds. We determine the $d$-relaxed game chromatic number for classes $\mathcal{H}_{k}(k \in\{3,4\})$, proving that whenever $k \in\{3,4\}$ and $d \in\{1, \ldots, k-1\}$, then $\chi_{g}^{(d)}\left(\mathcal{H}_{k}\right) \leq k+2-d$.

Although there are many results concerning the game chromatic number, there are very few strategies to play the colouring game. The most known strategy is the Activation strategy that was introduced for trees in [10]. Then it was simplified in [13]. Until this it was used for the colouring game in many papers. The modification of this strategy was used for relaxed colouring games [7,8], for asymmetric games [18,19], for oriented colouring games [14]. In this paper, we propose the new strategy to play the colouring game and the relaxed colouring game. The idea of this strategy is based on a unilateral game in which Bob is more powerful than Alice, he has more freedom in colour selection and can miss his move. More precisely, let be given a graph $G$, an additive hereditary property $\mathcal{P}$ and a set $C$ of $r$ colours. Similarly as above two players Alice and Bob colour vertices of $G$. Alice starts the game. The players play alternately, but Bob can miss his move. Bob can colour the vertex with an arbitrary colour from $C$, while Alice must colour the vertex with a colour from $C$ in such a way that she cannot create a monochromatic minimal forbidden subgraph for the property $\mathcal{P}$. If after $|V(G)|$ moves all vertices of the graph $G$ are coloured, then Alice wins the game, otherwise Bob wins. Thus, Bob wins the game if after a move of some player there is an uncoloured vertex $v$ such that after colouring $v$ with any colour $i \in C$ a monochromatic minimal forbidden subgraph for $\mathcal{P}$ appears. The above game is called the unilateral $\mathcal{P}$-colouring game. The $\mathcal{P}$-unilateral game chromatic number, denoted 
by $\chi_{u g}^{\mathcal{P}}(G)$, is the least number $r$ such that Alice has a winning strategy, when playing the unilateral $\mathcal{P}$-colouring game with $r$ colours on $G$.

In general, the game chromatic numbers are not monotone, in Sect. 2 we give the example showing that the $\mathcal{P}$-game chromatic number is not monotone. However, the unilateral game chromatic number is monotone, we prove this in Sect. 3. In Sect. 3 we also give other interesting properties of the unilateral game chromatic number and the unilateral game. We show that if Alice can win the unilateral game with $r$ colours on $G$, then she also can win the unilateral game with more than $r$ colours what gives the partial solution for the problem stated by Zhu [20]. Moreover, we prove that the unilateral game chromatic number is an upper bound for the game chromatic number and the relaxed game chromatic number, i.e., $\chi_{g}(G) \leq \chi_{g}^{\mathcal{O}}(G), \chi_{g}^{(d)}(G) \leq \chi_{g}^{\mathcal{P}}(G)$, where $\mathcal{O}$ denotes the class of edgeless graphs. In Sect. 4 give an effective strategy for playing the unilateral game. In Sect. 5 we introduce the notation that we need in Sects. 6 and 7. Let $\mathcal{S}_{d}$ denote the class of graphs with maximum degree at most $d$. In Sects. 6 and 7 we study the $\mathcal{S}_{d}$-unilateral game chromatic number $(d \in\{2,3\})$ for graphs from $\mathcal{H}_{3}$ and $\mathcal{H}_{4}$, respectively. In Sect. 8, we use the $\mathcal{S}_{d}$-unilateral game chromatic number to obtain the upper bound for $d$-relaxed game chromatic number, namely we obtain that for $k \in\{3,4\}$ and $0 \leq d \leq k-1, \chi_{g}^{(d)}\left(\mathcal{H}_{k}\right) \leq k+2-d$.

\section{The Example of the non-Monotonicity of the $\mathcal{P}$-Game Chromatic Number}

For many known graphs $G$ the following holds: $\chi_{g}\left(G^{\prime}\right) \leq \chi_{g}(G)$ for any subgraph $G^{\prime}$ of $G$. However, the game chromatic number is not monotone, i.e., from the fact that Alice has a winning strategy for the colouring game on $G$ it does not follow that Alice has a winning strategy on a proper subgraph of $G$. The well-known example is the complete bipartite graph $K_{n, n}(n \geq 4)$. It is easy to see that $\chi_{g}\left(K_{n, n}\right)=3$. Hence, Alice has a winning strategy for the 3-colouring game on $K_{n, n}$. However, Bob has a winning strategy for the 3-colouring game on $G=K_{n, n}-M$, where $M$ is a perfect matching of $K_{n, n}$. In this section we give an example showing that the generalized game chromatic number is not monotone. Recall that $\mathcal{T}_{1}$ denotes the class of forests. We consider the $\mathcal{T}_{1}$-game chromatic number of $K_{n, n} \cup K_{1}$, its induced subgraph $K_{n, n}$ and its subgraph $K_{n, n}-M$ where $M$ is a perfect matching of $K_{n, n}$. We show that $\chi_{g}^{\mathcal{T}_{1}}\left(K_{n, n} \cup K_{1}\right)<\chi_{g}^{\mathcal{T}_{1}}\left(K_{n, n}\right)<\chi_{g}^{\mathcal{T}_{1}}\left(K_{n, n}-M\right)$. In this section the vertices of $K_{n, n}$ are denoted by $V=\left\{v_{1}, \ldots, v_{n}\right\}$ and $W=\left\{w_{1}, \ldots, w_{n}\right\}$ where $V$ and $W$ are two independent sets of $K_{n, n}$.

Proposition 1 For $n \geq 2$, it holds $\chi_{g}^{\mathcal{T}_{1}}\left(K_{n, n} \cup K_{1}\right)=2$.

Proof It is easy to see that $\chi_{g}^{\mathcal{T}_{1}}\left(K_{n, n} \cup K_{1}\right) \geq 2$. Moreover, $\chi_{g}^{\mathcal{T}_{1}}\left(K_{n, n} \cup K_{1}\right)=2$ for $n=2$. We show that Alice wins the $\mathcal{T}_{1}$-colouring game with two colours on $K_{n, n} \cup K_{1}$ for $n \geq 3$. Let $C=\{1,2\}$ be the set of colours. Alice in the first move colours an isolated vertex with any colour. Next, observe the following.

Claim If after some moves of the players in $V$ there are two vertices coloured with 1 and in $W$ there are two vertices coloured with 2, then Alice wins the game. 

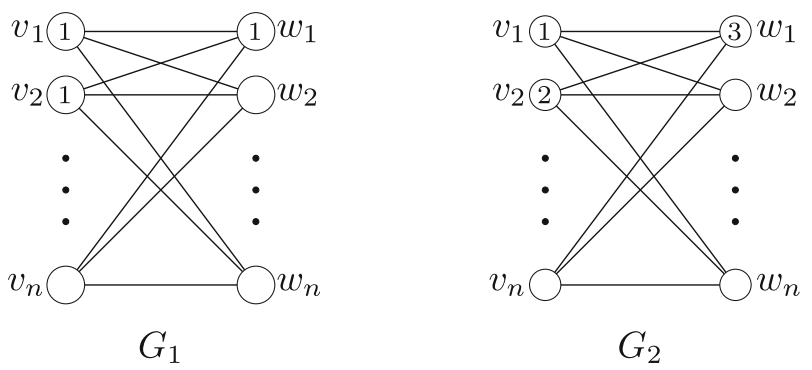

Fig. 1 The structures in Proposition 2

Proof Since during the game players cannot create any monochromatic cycle, in $V$ there is at most one vertex coloured with 2 and in $W$ there is at most one vertex coloured with 1 . Thus, each vertex of $V$ can be coloured with 1 and each vertex of $W$ can be coloured with 2 and if the players continue the game, the colour 1 will be always admissible for each uncoloured vertex of $V$ and the colour 2 will be always admissible for each uncoloured vertex of $W$.

Assume without loss of generality that in his first move Bob colours $v_{1}$ with 1 . Then, Alice colours the vertex $w_{1}$ with 1 . If in the second move Bob colours a vertex with 1, then Alice colours a vertex with 2 in the opposed set. In the next move she colours a vertex with 2 in the same set, so she wins. If in the second move Bob colours a vertex with 2, then Alice colours a vertex in the opposed set with 1. And similarly as above in the next move she colours a vertex with 2 in the set that does not contain two vertices with 1 .

Proposition 2 For $n \geq 4$, it holds $\chi_{g}^{\mathcal{T}_{1}}\left(K_{n, n}\right)=3$.

Proof First, we show that Bob wins the $\mathcal{T}_{1}$-colouring game with two colours on $K_{n, n}$. Let $C=\{1,2\}$ be the set of colours. Observe that if after some moves of the players in $V$ there are two vertices coloured with 1 and two vertices coloured with 2, then Bob wins the game, since at most two vertices of $W$ can be coloured. The same holds for the set $W$. Assume without loss of generality that in her first move Alice colours $v_{1}$ with 1 . Then, Bob colours the vertex $v_{2}$ with 2 . In his next moves Bob plays such that he results in two vertices coloured with 1 and two vertices coloured with 2 in $V$. It is easy to see that Bob can achieve such a goal.

Now, we show that Alice wins the $\mathcal{T}_{1}$-colouring game with three colours on $K_{n, n}$. Let $C=\{1,2,3\}$ be the set of colours. Similarly as in Proposition 1 we can show that if after some moves of the players in $V$ there are two vertices coloured with 1 and in $W$ there are two vertices coloured with 2, then Alice wins the game. Observe that because of the symmetry of colours the same holds for any other pair of colours.

Claim If after three moves of players one of the structures presented in Fig. 1 appears, then Alice wins the game.

Proof Suppose that $G_{1}$ appears. Alice's goal is to get two vertices coloured with 2 (or with 3 ) in the set $W$. Now, it is Bob's move. Suppose that Bob colours a vertex of $W$. He cannot colour it with colour 1, so he uses the colour 2 or 3 . Alice colours the other 
vertex of $W$ with the same colour and hence she wins. If Bob colours a vertex of $V$ with 1 , then Alice colours a vertex of $W$ with 2 and in the next move she achieves the goal. If Bob colours a vertex of $V$ with 2, then Alice colours a vertex of $W$ with 3 . In the next move she colours a vertex of $W$ with 3 and she wins. The case is similar when Bob colours a vertex of $V$ with 3 .

Now, suppose that $G_{2}$ appears. In her next move Alice colours an uncoloured vertex of $W$ with 3 , so after her move in $W$ there are two vertices coloured with 3 . The next Alice's move depends on two previous moves of Bob. But she always results in two vertices coloured with one colour (either 1 or 2 ) in $V$.

The claim remains true if in the structures we replace colours symmetrically. The observation that Alice can play in such a way that one of the structures presented in Fig. 1 appears, completes the proof.

Proposition 3 For $n \geq 8$, it holds $\chi_{g}^{\mathcal{T}_{1}}\left(K_{n, n}-M\right)>3$.

Proof Let $M=\left\{v_{1} w_{1}, \ldots, v_{n} w_{n}\right\}$. We show that Bob wins the $\mathcal{T}_{1}$-colouring game with three colours on $K_{n, n}-M$. Let $C=\{1,2,3\}$ be the set of colours. First, observe the following.

Claim If after some moves of the players $G$ contains at least three uncoloured vertices in $V$, and at least three uncoloured vertices in $W$, and there are two monochromatic paths $P_{4}$ coloured with two distinct colours, then Bob wins the game.

Proof Since $G$ cannot contain a monochromatic cycle, these two paths are induced subgraphs of $G$. Moreover, two vertices of each path are in $V$ and two other are in $W$. Assume without loss of generality that one path is coloured with 1 and the other with 2. Thus, the uncoloured vertices can be coloured neither with 1 nor with 2 . However, the uncoloured vertices induces subgraph with a cycle, so Alice needs at least two new colours to win the game.

Observe that Bob has a strategy resulting in two monochromatic $P_{4}$ coloured with two distinct colours. The main idea of this strategy is the following: after each Bob's move the set $T$ of vertices coloured with colour $t(t \in\{1,2,3\})$ satisfies:

(i) $|T|=4$ and $T$ induces the path $P_{4}$, or

(ii) $T=\left\{v_{i}, w_{i}\right\}$ or $T=\left\{v_{i}, w_{j}\right\}(i \neq j)$ such that $w_{i}$ and $v_{j}$ are coloured, or

(iii) $T=\varnothing$.

Using this strategy after at most 5 moves of each player Bob achieves the goal, i.e., two monochromatic $P_{4}$ coloured with two distinct colours appear. Let us consider some first moves of the players. If Alice colours $v_{1}$ with 1 , then Bob colours $w_{1}$ with 1. If in the second move Alice colours $v_{2}$ with 2, then Bob colours $w_{2}$ with 2. If in the second move Alice colours $v_{2}$ with 1 , Bob colours $w_{3}$ with 1 , to create the monochromatic $P_{4}$. If next Alice colours $w_{2}$ (or $v_{3}$ ) with 2, then Bob colours $v_{3}$ (or $w_{2}$ ) with 2 . Next, Bob continues in the same way.

To complete the proof observe that if Bob uses the same strategy for playing the $\mathcal{T}_{1}$-colouring game with two colours, then he wins. 


\section{Properties of the Unilateral $\mathcal{P}$-Colouring Game}

The unilateral $\mathcal{P}$-colouring game and the $\mathcal{P}$-unilateral game chromatic number are interesting because of their special properties. As we see in Sect. 2 the $\mathcal{P}$-game chromatic number is not monotone which often causes difficulties in proofs. Next, lemmas give the monotone properties of the $\mathcal{P}$-unilateral game chromatic number.

Lemma 1 If $G^{\prime}$ is a spanning subgraph of $G$, then $\chi_{u g}^{\mathcal{P}}\left(G^{\prime}\right) \leq \chi_{u g}^{\mathcal{P}}(G)$.

Proof In the unilateral $\mathcal{P}$-colouring game on $G^{\prime}$, Alice colours the vertices such that she does not create in $G$, a monochromatic minimal forbidden subgraph for $\mathcal{P}$, so she plays on $G^{\prime}$ but she sees $G$. She can achieve such a goal, since she has a winning strategy for the unilateral $\mathcal{P}$-colouring game on $G$.

Lemma 2 If $G^{\prime}$ is a induced subgraph of $G$, then $\chi_{u g}^{\mathcal{P}}\left(G^{\prime}\right) \leq \chi_{u g}^{\mathcal{P}}(G)$.

Proof Let $\chi_{u g}^{\mathcal{P}}(G)=r$. Suppose that Bob has a winning strategy for the unilateral $\mathcal{P}$-colouring game with $r$ colours on $G^{\prime}$. If Bob plays on $G$ using this strategy in such a way that he misses the move when Alice colours a vertex of $G \backslash G^{\prime}$ (he waits until Alice colours a vertex of $G^{\prime}$ to make a move according to his strategy on $G^{\prime}$ ), then Bob wins this game, a contradiction.

From Lemmas 1 and 2 it immediately follows

Lemma 3 If $G^{\prime}$ is a subgraph of $G$, then $\chi_{u g}^{\mathcal{P}}\left(G^{\prime}\right) \leq \chi_{u g}^{\mathcal{P}}(G)$.

In Zhu [20] suggested that if $\chi_{g}(G)=r$, then Alice has a winning strategy for the $t$-colouring game on $G$ for any $t \geq r$. The other property of the unilateral $\mathcal{P}$-colouring game gives the partial solution to this problem.

Lemma 4 If Alice has a winning strategy for the unilateral $\mathcal{P}$-colouring game on $G$ with $r$ colours, then she also has a winning strategy for the unilateral $\mathcal{P}$-colouring game on $G$ with $t$ colours for any $t \geq r$.

Proof Since Alice has a winning strategy for the unilateral $\mathcal{P}$-colouring game on $G$ with $r$ colours, during the game after each move for any uncoloured vertex at least one colour from $\{1, \ldots, r\}$ is admissible. Observe that using by Bob the colour $c>r$ does not result in impasse on any vertex. Thus, also in the game with $t$ colours after each move for any uncoloured vertex at least one colour from $\{1, \ldots, r\}$ is admissible.

In the unilateral colouring games Bob is more powerful than in the ordinary colouring games, this implies the following

Lemma 5 If Alice has a winning strategy for the unilateral $\mathcal{P}$-colouring game on $G$ with $r$ colours, then she also has a winning strategy for the $\mathcal{P}$-colouring game on $G$ with $r$ colours.

From Lemma 5 it follows that the unilateral $\mathcal{P}$-colouring game can be used as the tool in the study of the $\mathcal{P}$-colouring game. In many papers, the other invariant was used 
to determine the game chromatic number-the game colouring number, which was introduced in [20]. It is also defined as a two person game-the marking game. Alice and Bob, with Alice moving first, alternately mark an unmarked vertex. The game ends when all vertices are marked. For each vertex $v \in V(G)$, let $s(v)$ denote the number of neighbours of $v$ that are marked before $v$. Let $s=1+\max _{v \in V} s(v)$ be the score of the game. Alice's goal is to minimize the score, while Bob's goal is to maximize it. The game colouring number $\operatorname{col}_{g}(G)$ is the least $s$ such that Alice has a strategy that results in a score at most $s$. It is easy to see that for any graph $G, \chi_{g}(G) \leq \operatorname{col}_{g}(G)$. The next lemma shows that the unilateral game chromatic number gives a better bound for the game chromatic number. Recall that $\mathcal{O}$ denotes the class of edgeless graphs.

Lemma 6 For any graph $G$, it holds $\chi_{g}(G) \leq \chi_{u g}^{\mathcal{O}}(G) \leq \operatorname{col}_{g}(G)$.

Proof Since the $\mathcal{O}$-colouring game with $r$ colours is exactly the same as the $r$-colouring game, by Lemma 5 we have $\chi_{g}(G) \leq \chi_{u g}^{\mathcal{O}}(G)$. Now, we show that $\chi_{u g}^{\mathcal{O}}(G) \leq \operatorname{col}_{g}(G)$. To start with, let us define a unilateral marking game. In the unilateral marking game Bob can miss his move, the remaining rules of the game are the same as in the marking game. Observe that missing the move by Bob does not help him to maximize the score in the unilateral marking game. Thus, to play optimally in the unilateral marking game Bob will not miss the move. From this it follows that if Alice has a winning strategy for the marking game with score $r$, then she also has a winning strategy for the unilateral marking game with score $r$. Note that in the unilateral marking game with score $r$ after each move any unmarked vertex has at most $r-1$ marked neighbours. In the unilateral $\mathcal{O}$-colouring game Alice first chooses a vertex to colour according to her winning strategy for the unilateral marking game with score $r$, next she colours the vertex with a colour distinct from the colour of its neighbours. She can always find such a colour, since the vertex has at most $r-1$ coloured neighbours. Hence, Alice wins the game with $r$ colours, so $\chi_{u g}^{\mathcal{O}}(G) \leq \operatorname{col}_{g}(G)$.

The unilateral colouring game can also be used as the tool in the study of the relaxing colouring game. Since the $\mathcal{S}_{d}$-colouring game with $r$ colours is exactly the same as the $(r, d)$-relaxed colouring game, by Lemma 5 we have the following

Lemma 7 For any graph $G$, it holds $\chi_{g}^{d}(G) \leq \chi_{u g}^{\mathcal{S}_{d}}(G)$.

It is easy to see that Alice has the winning strategy for the 3-colouring game on $K_{n, n}$ (the $(3,0)$-relaxed colouring game). However, one can observe that Bob has the winning strategy for the $(3,1)$-relaxed colouring game on $K_{n, n}$. For $d \geq 1$, we do not know whether Alice can win the $(r, d+1)$-relaxed colouring game when she can win the $(r, d)$-relaxed colouring game. The next lemma gives the answer to this question for the unilateral $\mathcal{S}_{d}$-colouring.

Lemma 8 Let $d \geq 0$. Then $\chi_{u g}^{\mathcal{S}_{t}}(G) \leq \chi_{u g}^{\mathcal{S}_{d}}(G)$ for every $t \geq d$.

Proof Let $\chi_{u g}^{\mathcal{S}_{d}}(G)=k$. Thus, Alice has a strategy to win the unilateral $\mathcal{S}_{d}$-colouring game with $k$ colours. The property $\mathcal{S}_{d}$ has exactly one minimal forbidden subgraph $\mathbf{F}\left(\mathcal{S}_{d}\right)=\left\{K_{1, d+1}\right\}$. If a vertex is not contained in any subgraph $K_{1, d+1}$, then it is 
also not contained in any subgraph $K_{1, t+1}$ for every $t \geq d$. Thus, if Alice colours the vertices such that she does not create a monochromatic $K_{1, d+1}$, then she also does not create any monochromatic $K_{1, t+1}$ for every $t \geq d$, so she wins the unilateral $\mathcal{S}_{t}$-colouring game with $k$ colours. Hence, $k \geq \chi_{u g}^{\mathcal{S}_{t}}(\bar{G})$.

Lemmas 5 and 8 imply

Lemma 9 Let $d \geq 1$. Then $\chi_{g}^{t}(G) \leq \chi_{u g}^{\mathcal{S}_{d}}(G)$ for every $t \geq d$.

\section{Strategy for the Unilateral $\mathcal{P}$-Colouring Game}

In this section we present an effective strategy for Alice to play the unilateral $\mathcal{P}$ colouring game. We start with some notations.

Let $G$ be a graph, $C=\{1, \ldots, r\}$ and $S \subseteq V(G)$. A partial $r$-colouring of $G$ is a function $c_{r}: S \rightarrow C$. The pair $\left(G, c_{r}\right)$ is called a partially $r$-coloured graph. If the graph is uncoloured or all vertices of the graph are coloured, then we also say that the graph is partially $r$-coloured. Let $\mathcal{G}^{r}$ denote a family of partially $r$-coloured graphs $\left(G, c_{r}\right)$.

Let $\mathcal{P}$ be the additive hereditary property. Let $G$ be a partially $r$-coloured graph and $v$ be an uncoloured vertex of $G$. We say that colour $i$ is $(\mathcal{P}, r)$-admissible for $v$ if $i \in\{1 \ldots, r\}$ and after colouring $v$ with $i$ no monochromatic minimal forbidden subgraph from $\mathbf{F}(\mathcal{P})$ containing $v$ appears. If $\mathcal{P}$ and $r$ are clear from the context, we may omit them.

From definitions it immediately follows

Lemma 10 If Alice has on $G$ the strategy for the unilateral $\mathcal{P}$-colouring game with $r$ colours such that after each move of any player there is a $(\mathcal{P}, r)$-admissible colour for each uncoloured vertex, then Alice wins the game.

Let $G$ be a graph and $\gamma(G)$ be a family of partially $r$-coloured graphs, i.e., $\gamma(G) \subseteq \mathcal{G}^{r}$.

Definition $1((\mathcal{P}, r)$-game closed family) The family $\gamma(G)$ is $(\mathcal{P}, r)$-game closed if for every graph $\left(G, c_{r}\right) \in \gamma(G)$ the following conditions hold:

(i) If in $\left(G, c_{r}\right)$ there are uncoloured vertices, then there is a vertex that can be coloured by Alice with a $(\mathcal{P}, r)$-admissible colour in such a way that the resulting graph $\left(G, c_{r}^{\prime}\right)$ is in $\gamma(G)$.

and

(ii) If Bob colours a vertex of $\left(G, c_{r}\right)$ with an arbitrary colour $i, i \in\{1, \ldots, r\}$ and after his move there are still uncoloured vertices, then there is a vertex that can be coloured by Alice with a $(\mathcal{P}, r)$-admissible colour in such a way that the resulting graph $\left(G, c_{r}^{\prime \prime}\right)$ is in $\gamma(G)$.

Example 1 To illustrate Definition 1 we construct the $(\mathcal{O}, 4)$-game closed family for a tree. Let $T$ be an arbitrary tree. Each component of $T-v$ is called stem of $v$. A coloured stem of $v$ is a stem of $v$ with at least one coloured vertex. Let $\gamma(T) \subseteq \mathcal{T}^{4}$ be 
the family of partially 4-coloured trees such that $\left(T, c_{4}\right) \in \gamma(T)$ if each uncoloured vertex has at most two coloured stems. One can observe that $\gamma(T)$ is $(\mathcal{O}, 4)$-game closed: if $\left(T, c_{4}\right) \in \gamma(T)$, then Alice can colour a vertex in such a way that resulting tree is in $\gamma(T)$; if Bob colours a vertex, then at most one vertex with three coloured stems may appear, thus Alice colours this vertex and the resulting tree is in $\gamma(T)$.

Definition $2((G ; \mathcal{P}, r)$-strategy)

1. Alice creates a $(\mathcal{P}, r)$-game closed family $\gamma(G)$ containing the uncoloured graph $G$.

2. Alice plays in such a way that each time after her move the resulting graph $\left(G, c_{r}\right)$ belongs to $\gamma(G)$.

Remark 1 The $(G ; \mathcal{P}, r)$-strategy exists if and only if the $(\mathcal{P}, r)$-game closed family containing the uncoloured $G$ exists.

First, observe the following

Lemma 11 Let $\gamma(G)$ be a $(\mathcal{P}, r)$-game closed family. If $\left(G, c_{r}\right) \in \gamma(G)$, then for any uncoloured vertex $u$ there exists a colour that is $(\mathcal{P}, r)$-admissible.

Proof Assume that in $\gamma(G)$ there is a graph that has an uncoloured vertex $u$ with no $(\mathcal{P}, r)$-admissible colour. Let $\left(G, c_{r}\right)$ be the graph with such a vertex and with the minimum number of uncoloured vertices. If $u$ is the only uncoloured vertex, then $\left(G, c_{r}\right)$ does not satisfy Condition (i) of Definition 1, a contradiction. If ( $\left.G, c_{r}\right)$ has more than one uncoloured vertex, then Bob can colour a vertex other than $u$. By Condition (ii) of Definition 1 there is a vertex that can be coloured by Alice with the $(\mathcal{P}, r)$-admissible colour in such a way that the resulting graph $\left(G, c_{r}^{\prime}\right)$ is in $\gamma(G)$. Thus, after her move a vertex $u$ is uncoloured and has no $(\mathcal{P}, r)$-admissible colour, and $\left(G, c_{r}^{\prime}\right)$ has fewer uncoloured vertices, a contradiction.

Theorem 3 Let $\gamma(G)$ be a $(\mathcal{P}, r)$-game closed family and $G \in \gamma(G)$. Assume that Alice and Bob play the unilateral $\mathcal{P}$-colouring game with $r$ colours on $G$. If Alice uses the $(G ; \mathcal{P}, r)$-strategy, then she wins the game.

Proof From Definition 1 it follows that Alice can play in such a way that after her move $\left(G, c_{r}\right)$ is in $\gamma(G)$. By Lemma 11 after each move of any player every uncoloured vertex of $\left(G, c_{r}\right)$ has a $(\mathcal{P}, r)$-admissible colour. Thus, by Lemma 10 Alice wins.

From Example 1 it follows that there is $(\mathcal{O}, 4)$-game closed family $\gamma(T)$ for every tree $T$. Thus, Alice can use the $(T ; \mathcal{O}, 4)$-strategy for playing the unilateral $\mathcal{O}$-game on $T$ with four colours, i.e., she plays such that after her move the partially coloured T belongs to $\gamma(T)$. So $\chi_{u g}^{\mathcal{O}}(T) \leq 4$. Since $\chi_{g}(G) \leq \chi_{u g}^{\mathcal{O}}(G)$ for every graph $G$, we obtain the known result $\chi_{g}(T) \leq 4$ for every tree $T$.

Similarly as Theorem 3 we can prove the next theorem.

Theorem 4 Let $\gamma(G)$ be a $(\mathcal{P}, r)$-game closed family and $G \in \gamma(G)$. Assume that Alice and Bob play the unilateral $\mathcal{P}$-colouring game with $r$ colours on $G$, with the first move of Bob. If Alice uses the $(G ; \mathcal{P}, r)$-strategy, then she wins the game. 


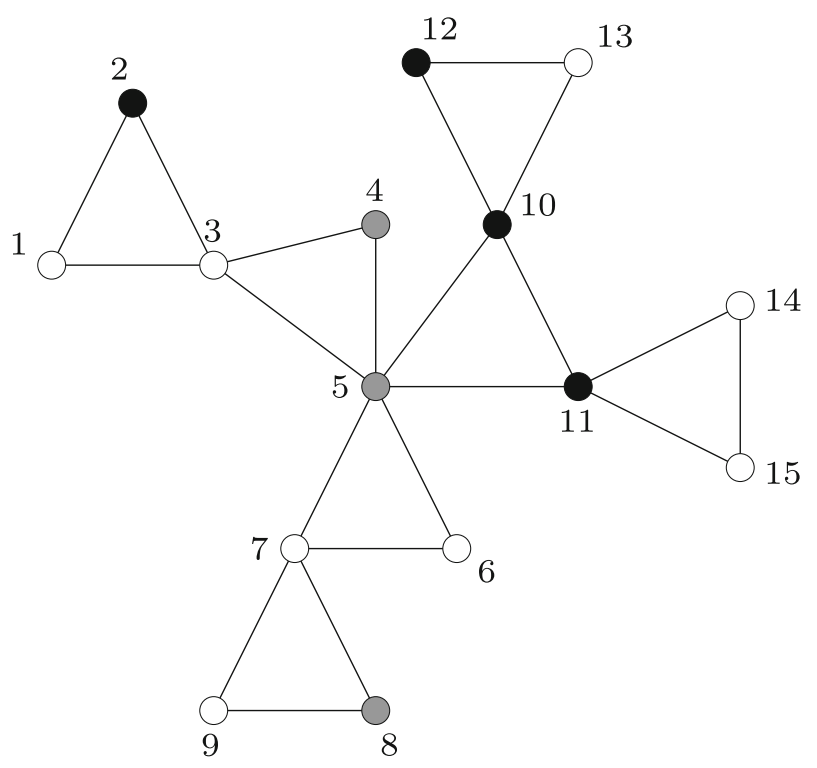

Fig. 2 The partially 2-coloured graph $G$ in Example 2

Previous results imply a very useful property of the $(G ; \mathcal{P}, r)$-strategy.

Corollary 1 Let $G=G_{1} \cup G_{2} \cup \ldots \cup G_{n}$. If there exists the (P, $\left.r\right)$-game closed family containing $G_{i}$ for $i \in\{1, \ldots n\}$, then Alice has the winning strategy for the unilateral $\mathcal{P}$-colouring game with $r$ colours on $G$.

Proof From Theorem 3 it follows that Alice has a winning strategy for the unilateral $\mathcal{P}$-colouring game with $r$ colours on each graph $G_{i}$, by Theorem 4 we have that Alice has a winning strategy for the same game and with the first move of Bob on any $G_{i}$, these imply that Alice has a winning strategy for the game on $G$.

\section{Notation}

Let $G \in \mathcal{H}_{k}$ and $G$ be a connected partially $r$-coloured graph. Recall that $G \in \mathcal{H}_{k}$ if every block of $G$ has at most $k$ vertices.

Let $u, v \in V(G)$. A separator of $u$ and $v$ is a vertex $w$ such that vertices $u$ and $v$ are in distinct components of $G-w$. Each component of $G-v$ is called a stem of $v$. If $v$ is not a cut-vertex of $G$, then it has exactly one stem. A stem of $u$ that has at least one coloured vertex is called a coloured stem of $u$. A stem of $u$ whose all vertices are uncoloured is called an uncoloured stem of $u$.

A graph with a monochromatic center is a graph with at least one coloured vertex in which a maximal connected monochromatic subgraph is fixed. This fixed connected monochromatic subgraph is called a monochromatic center of the graph.

Example 2 Let $G$ be a partially 2-coloured graph (see Fig. 2). Each maximal connected monochromatic subgraph of $G$ can be a monochromatic center of $G$. Thus, we can 
choose a subgraph induced by one of the sets: $\{4,5\},\{10,11,12\},\{8\}$, or $\{2\}$ to be a monochromatic center of $G$.

Next, definitions relate to the graph with a monochromatic center. Let $S$ be a monochromatic center of $G$. Let $B$ be a block of $G$ with at most one vertex in $S$. If the block $B$ has exactly one vertex in $S$, then a block-cut-vertex of $B$ is the vertex in $B \cap S$, otherwise a block-cut-vertex of $B$ is a vertex $w \in B$ such that in the graph $G-w$ vertices of $S$ and the remaining vertices of $B$ are in distinct components. If a block has more than one vertex in $S$, then it has no block-cut-vertex. Let $v \in V(G) \backslash S$. A back-stem of $v$ is a stem of $v$ containing $S$. A front-stem of $v$ is a stem of $v$ that does not contain $S$. A back-block of $v$, denoted by $B_{b}(v)$, is a block containing $v$ and the vertices of the back-stem of $v$. Note that every vertex has exactly one back-block (back-stem). Let $B_{b}^{\prime}(v)=B_{b}(v) \backslash T$, where $T$ is a block-cut-vertex of $B_{b}(v)$ or $S$ whenever $B_{b}(v)$ has no block-cut-vertex. A front-block of $v$ is a block containing $v$ and the vertices of a front-stem of $v$. The family of all front-blocks of $v$ we denote by $\mathcal{B}_{f}(v)$. The vertex $v$ is a block-cut-vertex of every block from $\mathcal{B}_{f}(v)$. A backneighbour (front-neighbour) of $v$ is a neighbour of $v$ belonging to the back-block (a front-block) of $v$. The set of vertices that are front-neighbours (back-neighbours) of $v$ we denote by $N_{f}(v)\left(N_{b}(v)\right)$, respectively.

The vertices $v$ and $u$ are similar if they are coloured and $c(v)=c(u)$. In the proofs the vertices that have similar front-neighbours play an important role.

Example 3 Let $G$ be a partially 2-coloured graph with the monochromatic center $S=\{2,3,5\}$ (see Fig. 3). Each triangle is a block of $G$. The block $\{3,4,5\}$ has no block-cut-vertex, since it contains two vertices of $S$, similarly the block $\{1,2,3\}$. All other blocks have the block-cut-vertex. For example, for the block $\{4,6,7\}$ the vertex 4 is the block-cut-vertex, the vertex 6 is the block-cut-vertex of two blocks: $\{6,8,9\}$ and $\{6,12,13\}$, the vertex 5 is the block-cut-vertex of $\{5,16,17\}$. The vertex 4 has exactly one front-stem $\{6, \ldots, 15\}$ and exactly one back-stem $\{1,2,3,5\} \cup$ $\{16, \ldots, 23\} . B_{b}(4)=\{3,4,5\}$ is the back-block of 4 . Since $B_{b}(4)$ has no cut-blockvertex, $T=S$, so $B_{b}^{\prime}(4)=\{4\}$. The vertex 4 has the exactly one front-block $\mathcal{B}_{f}(4)=$ $\{4,6,7\}$. The vertex 6 has two front-stems: $\{8,9,10,11\}$ and $\{12,13,14,15\}$. The remaining vertices of $G-\{6\}$ form the back-stem of $6 . B_{b}(6)=\{4,6,7\}$ is the back-block of 6 and $B_{b}^{\prime}(6)=\{6,7\}$. The vertex 6 has two front-blocks, $\mathcal{B}_{f}(6)=$ $\{\{6,8,9\},\{6,12,13\}\}$. The vertex 19 has neither a front-stem nor a front-block. All vertices of $G-\{19\}$ form the back-stem and hence $B_{b}(19)=\{1,18,19\}, B_{b}^{\prime}(19)=$ $\{18,19\}$.

\section{The Unilateral $\mathcal{S}_{2}$-Colouring Game on Graphs from $\mathcal{H}_{3}$}

We prove that Alice wins the unilateral $\mathcal{S}_{2}$-colouring game with three colours on any graph from $\mathcal{H}_{3}$. Thus, we prove the following

Theorem 5 Every graph $G \in \mathcal{H}_{3}$ satisfies $\chi_{u g}^{\mathcal{S}_{2}}(G) \leq 3$.

The main part of this proof is the construction of the $\left(\mathcal{S}_{2}, 3\right)$-game closed family for any uncoloured graph $G \in \mathcal{H}_{3}$. Thus, Alice can use the $\left(G ; \mathcal{S}_{2}, 3\right)$-strategy to win the 


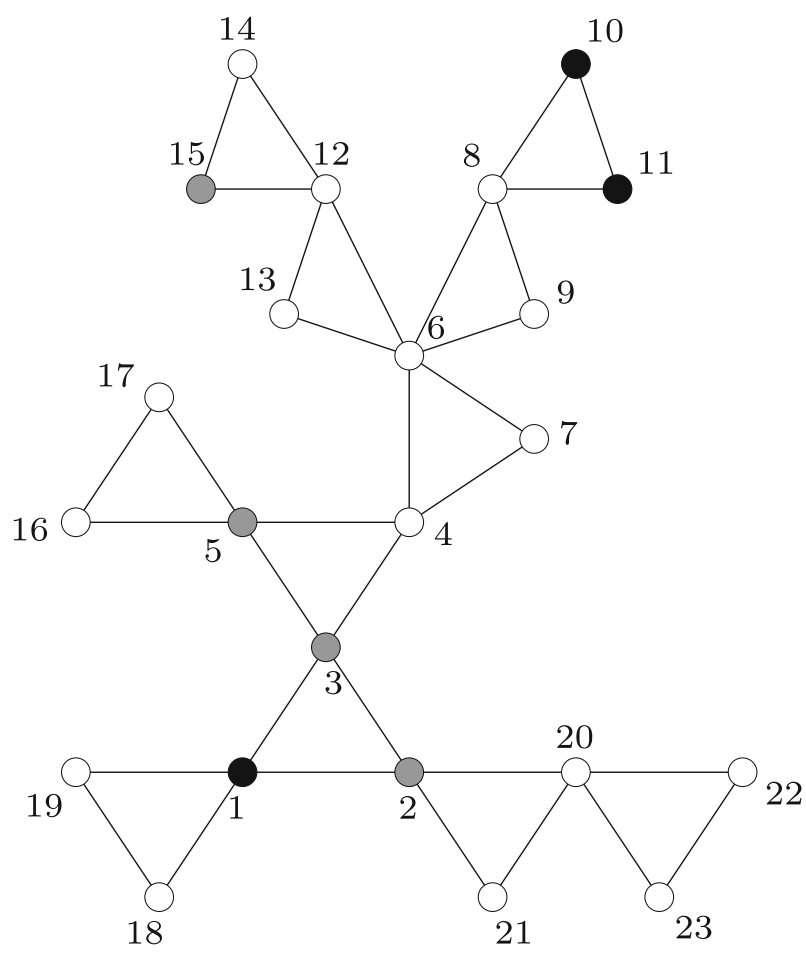

Fig. 3 The partially 2-coloured graph $G$ with the monochromatic center $S=\{2,3,5\}$ in Example 3

game with three colours. Since by Lemma 3 the $\mathcal{S}_{2}$-unilateral game chromatic number is monotone, we may assume that $G$ is connected and each block of $G$ is isomorphic to $K_{3}$.

Let $G \in \mathcal{H}_{3}$ and $G$ be a connected graph such that each block of $G$ is isomorphic to $K_{3}$.

Definition 3 (Family $\alpha_{2}(G)$ ) The family $\alpha_{2}(G)$ contains the uncoloured graph $G$ and the partially 3 -coloured graphs from $\mathcal{G}^{3}$ with the monochromatic center $S$ such that every uncoloured vertex $u$ has one of the following properties

1. $u$ has exactly one coloured stem, or

2. $u$ has exactly two coloured stems and

(a) let $v$ be the vertex in $B_{b}^{\prime}(u) \backslash\{u\}$, if $v$ is uncoloured, then it has exactly one coloured stem, if $v$ is coloured, then it has no similar front-neighbour,

(b) every coloured vertex of $\mathcal{B}_{f}(u)$ has at most one similar front-neighbour.

The following example illustrates Definition 3 and the idea of proof of Lemma 14.

Example 4 To prove that the family $\alpha_{2}(G)$ is $\left(\mathcal{S}_{2}, 3\right)$-game closed we must show that Conditions (i) and (ii) of Definition 1 hold. In the other words, for each $\left(G, c_{3}\right) \in \alpha_{2}(G)$ we must prove that Alice can colour a vertex of $\left(G, c_{3}\right)$ in such a way that after her move each uncoloured vertex of the resulting graph has either the property (1) or (2) 


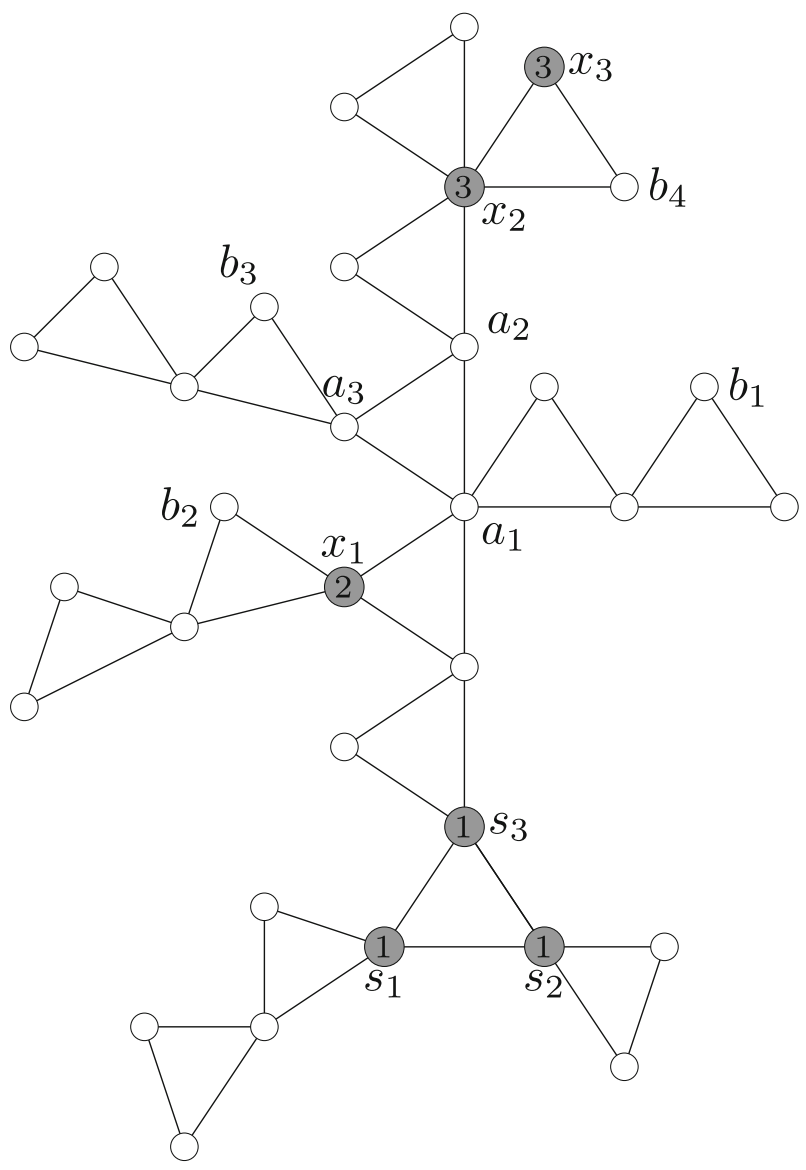

Fig. 4 The graph $G$ in Example 4

of Definition 3 and if Bob colours a vertex of $\left(G, c_{3}\right)$ and after his move there are uncoloured vertices, then there is a vertex that can be coloured by Alice in such a way that the resulting graph belongs to $\alpha_{2}(G)$. Let $G$ be uncoloured graph isomorphic to the graph in Fig. 4. Observe that a partially 3-coloured graph $\left(G, c_{3}\right)$ (see Fig. 4) belongs to $\alpha_{2}(G)$ : let us choose the vertices $\left\{s_{1}, s_{2}, s_{3}\right\}$ to be the monochromatic center $S$, then all uncoloured vertices have either the property (1) or (2); the uncoloured vertices on the shortest $\left(s_{3}-x_{2}\right)$-path have the property (2), the remaining uncoloured vertices have the property (1). Alice can colour an uncoloured neighbour of any vertex of $S$ with 2 , then the resulting graph is in $\alpha_{2}(G)$, so for $\left(G, c_{3}\right)$ the condition (i) of Definition 1 holds. Now, we consider some possible moves of Bob. Suppose that Bob colours the vertex $b_{1}$. After such a move the vertex $a_{1}$ has neither the property (1) nor (2), since $a_{1}$ has three coloured stems. Then, Alice colours $a_{1}$, she can use an arbitrary colour. If Bob colours $b_{2}$ with 2, then he destroys the property (2) of the vertex $a_{1}$ again, since in $B_{b}^{\prime}\left(a_{1}\right)$ there is the vertex $x_{1}$ having a similar front-neighbour. Thus, Alice colours $a_{1}$. Suppose that Bob colours $b_{3}$. Now, vertices $a_{2}$ and $a_{3}$ have neither 
the property (1) nor (2). In $B_{b}^{\prime}\left(a_{2}\right)$ and in $B_{b}^{\prime}\left(a_{3}\right)$ there is an uncoloured vertex with two coloured stems. Therefore, Alice colours either $a_{2}$ or $a_{3}$. If Bob colours $b_{4}$ with 3 , then in $B_{f}\left(a_{2}\right)$ there is the vertex $x_{2}$ with two similar front-neighbours. Hence, the vertex $a_{2}$ has neither the property (1) nor (2), so Alice colours $a_{2}$.

Next, two lemmas give some properties of graphs belonging to $\alpha_{2}(G)$.

Lemma 12 Let $G \in \alpha_{2}(G)$. Then for any uncoloured vertex there is at most one coloured front-stem.

Proof From Definition 3 it follows that any uncoloured vertex has at most two coloured stems. Since the back-stem of any vertex is coloured, there is at most one coloured front-stem.

Lemma 13 Let $G \in \alpha_{2}(G)$. Then in every block there are at most two uncoloured vertices with the property (2).

Proof First, we show that if in any block $B$ there are two vertices with the property (2), then one of them is the block-cut-vertex of $B$. Indeed, suppose that $u, w \in V(B)$ are two uncoloured vertices with the property (2) and neither $u$ nor $w$ is the block-cutvertex of $B$. Thus, $u$ is in the back-block of $w$ and $w$ is in the back-block of $u$ and hence $B_{b}^{\prime}(u) \backslash\{u\}=w$ and $B_{b}^{\prime}(w) \backslash\{w\}=u$. Condition 2(a) implies that $u$ has exactly one coloured stem and $w$ has exactly one coloured stem, so we have a contradiction. The observation that each block has at most one block-cut-vertex completes the proof.

Lemma 14 The family $\alpha_{2}(G)$ is $\left(\mathcal{S}_{2}, 3\right)$-game closed.

Proof Let $G \in \alpha_{2}(G)$. We show that Conditions (i) and (ii) of Definition 1 hold. Assume that Alice starts. If all vertices of $G$ are uncoloured, then Alice colours an arbitrary vertex. Thus, after Alice's move the coloured vertex is the monochromatic center $S$ and every uncoloured vertex of $G$ satisfies either the property (1) or the property (2). Suppose that $G$ has at least one coloured vertex. Thus, $G$ has the monochromatic center $S$ such that every uncoloured vertex of $G$ with $S$ satisfies either the property (1) or the property (2). We show that Alice can colour a vertex such that every uncoloured vertex of the resulting graph with the same center $S$ has the property (1) or the property (2). Let $B$ be a block with at least one uncoloured vertex such that $B$ has a coloured block-cut-vertex $w$ or has no a block-cut-vertex of $B$. Let $c_{s}=c(w)$ or $c_{s}=c(S)$, whenever a block-cut-vertex of $B$ does not exist. First, suppose that in $B$ there is a vertex $u$ with the property (2). Let $B_{f}(u)$ be a block of $\mathcal{B}_{f}(u)$ that is in the coloured front-stem. Note that if in $B_{f}(u)$ there is a vertex with two similar neighbours, then it has one similar neighbour in $B_{f}(u)$, so then in $B_{f}(u)$ there are two vertices coloured with one colour. If in $B_{f}(u)$ there are two vertices coloured with one colour, let us denote this colour by $c_{0}$. Alice colours $u$ with a colour such that $c(u)$ is distinct from $c_{s}$ and $c_{0}$. After Alice's move all vertices that had had the property (1) before Alice's move, still have the property (1) after her move and all vertices that had had the property (2) still have the property (2). Thus, $G \in \alpha_{2}(G)$. If in $B$ there is no vertex with the property (2), then Alice colours a vertex $u$ with the property (1). Alice colours $u$ with a colour distinct from $c_{s}$ and the colour of the third vertex in $B$. Now, we 
consider the case when Bob starts. If after his move $G \in \alpha_{2}(G)$, then obviously Alice can colour a vertex in such a way that also after her move $G$ is in $\alpha_{2}(G)$. Thus, assume that after Bob's move $G \notin \alpha_{2}(G)$. Before Bob's move every uncoloured vertex of $G$ with the monochromatic center $S$ had satisfied either the property (1) or the property (2), we show that after Bob's move Alice can colour a vertex such that again every uncoloured vertex of the resulting graph with the same center $S$ has the property (1) or the property (2). Let us consider two cases.

Case 1 Bob has coloured the vertex $b$ in such a way that he has destroyed the property (1) of the vertex $u$.

Thus, Bob has coloured a vertex of an uncoloured stem of $u$. Note, that if now the vertex $u$ has the property (2), then $G \in \alpha_{2}(G)$ : the vertices that are separators of $b$ and $u$ have the property (2), before Bob's move they had had the property (1); other vertices have the same property as they had had before Bob's move. So, we may assume that $u$ does not have the property (2). Let $\{v\}=B_{b}^{\prime}(u) \backslash\{u\}$. This implies that $v$ has two coloured stems if it is uncoloured or $v$ has a similar front-neighbour if it is coloured. Suppose that $B_{b}(u)$ has an uncoloured block-cut-vertex $w$. If $v$ is coloured, then Alice colours $u$ with an arbitrary colour. After such a move $w$ still has the property (2). If $v$ is uncoloured (it has the property 2), then Alice colours $u$ with a colour distinct from $c(b)$. After such a move vertices $w$ and $v$ still have the property (2). Suppose that $B_{b}(u)$ has a coloured block-cut-vertex $w$ or has no block-cut-vertex. Let $c_{s}=c(w)$ or $c_{s}=c(S)$, whenever a block-cut-vertex of $B_{b}(u)$ does not exist. Assume that $v$ is coloured. In this case colour $c_{s}$ and $c(v)$ may not be admissible for $u$, so Alice colours $u$ with a colour distinct from $c_{s}$ and $c(v)$. If $v$ is uncoloured, then Alice colours $u$ with a colour distinct from $c(b)$ and $c_{s}$. After Alice's move $v$ still has the property (2).

Case 2 Bob has coloured the vertex $b$ in such a way that he has destroyed the property (2) of the vertex $u$.

\section{Subcase 2.1 The vertex $u$ has three coloured stems.}

If $b$ is adjacent to $u$, then all uncoloured vertices of the stem of $u$ containing $b$ have the property (1). If $b$ is not adjacent to $u$, then vertices that are separators of $b$ and $u$ have the property (2) (before Bob's move they had had the property (1)) all other vertices of this stem have the property (1). Let $B_{f}(u)$ be a block of $\mathcal{B}_{f}(u)$ that is in the coloured front-stem not containing $b$. If in $B_{f}(u)$ there is a coloured vertex with two similar neighbours, then it has one similar neighbour in $B_{f}(u)$, so then in $B_{f}(u)$ there are two vertices coloured with the same colour.

First, suppose that in $B_{f}(u)$ there are two vertices coloured with the same colour, say $c_{0}$. If the block-cut-vertex $w$ of $B_{b}(u)$ is uncoloured, then Alice colours $u$ such that $c(u) \neq c_{0}$. After her move every coloured front-neighbour of $w$ has at most one similar front-neighbour, so $w$ has the property (2) and $G \in \alpha_{2}(G)$. Assume that the block-cut-vertex $w$ of $B_{b}(u)$ is coloured or does not exist. Let $c_{s}=c(w)$ or $c_{s}=c(S)$ (if $B_{b}(u)$ has no block-cut-vertex). In this case Alice colours $u$ with a colour distinct from $c_{0}$ and $c_{s}$, since these colours may not be admissible for $u$. 
Now, suppose that in $B_{f}(u)$ there are not any two vertices coloured with the same colour, i.e., the vertices of $B_{f}(u) \backslash\{u\}$ are coloured with distinct colours or at least one vertex of $B_{f}(u) \backslash\{u\}$ is uncoloured. Hence, every coloured vertex of $B_{f}(u)$ has at most one similar neighbour. If the block-cut-vertex $w$ of $B_{b}(u)$ is uncoloured, then Alice colours $u$ such that $c(u) \neq c(b)$. After Alice's move every coloured front-neighbour of $w$ has at most one similar front-neighbour, so $G \in \alpha_{2}(G)$. Otherwise, Alice colours $u$ in such a way that $c(u) \neq c(b)$ and $c(u) \neq c_{s}$, where $c_{s}$ is the colour of $w$ or the colour of the center. After Alice's move every uncoloured vertex has either the property (1) or the property (2).

Subcase 2.2 The vertex $v,\{v\}=B_{b}^{\prime}(u) \backslash\{u\}$, is coloured and has a similar frontneighbour.

Thus, Bob has coloured a front-neighbour of $v$. In this case $B_{b}(u)$ has a block-cutvertex $w$. Let $B_{f}(u)$ be the block of $\mathcal{B}_{f}(u)$ belonging to the coloured front-stem. Suppose that in $B_{f}(u)$ there are two vertices coloured with one colour, say $c_{0}$. Observe that then, only two colours may not be admissible for $u$ : $c_{0}$ and $c_{s}=c(w)$ (if vertex $w$ is coloured). Hence, Alice colours $u$ with a colour such that $c(u)$ is distinct from $c_{0}$ and $c_{s}$. If in $B_{f}(u)$ there are not any two vertices coloured with one colour, then Alice colours $u$ with a colour distinct from $c_{s}=c(w)$, whenever $w$ is coloured. After such a move $u$ has at most two similar neighbour and at most one similar front-neighbour. Hence, $G \in \alpha_{2}(G)$.

Subcase 2.3 The vertex $v,\{v\}=B_{b}^{\prime}(u) \backslash\{u\}$, is uncoloured and has two coloured stems.

Thus, Bob has coloured a vertex belonging to a front-stem of $v$. The vertex $v$ had had the property (1) before the Bob's move. Such a move was considered in Case 1.

Subcase $2.4 \operatorname{In} \mathcal{B}_{f}(u)$ there is a vertex with two similar front-neighbours.

Thus, Bob has coloured the front-neighbour $b$ of a coloured vertex of $\mathcal{B}_{f}(u)$. Note that if in $\mathcal{B}_{f}(u) \backslash\{u\}$ there is an uncoloured vertex, then it has the property (1). Alice colours $u$ with a colour such that $c(u)$ is distinct from $c(b)$ and $c_{s}$, where $c_{s}=c\left(B_{b}(u) \backslash B_{b}^{\prime}(u)\right)$.

Proof of Theorem 5 Let $G \in \mathcal{S}_{3}$. Let $G^{\prime}$ be a connected graph such that each block of $G^{\prime}$ is $K_{3}$ and $G \subseteq G^{\prime}$. Lemma 14 implies that there exists the $\left(\mathcal{S}_{2}, 3\right)$-game closed family containing $G^{\prime}$, so Alice has a winning strategy for the unilateral $\mathcal{S}_{2}$-game with three colours on $G^{\prime}$ (the $\left(G ; \mathcal{S}_{2}, 3\right)$-strategy). Hence, she plays in such a way that after her move the partially 3-coloured graph $G^{\prime}$ always belongs to $\alpha_{2}\left(G^{\prime}\right)$. Thus, $\chi_{u g}^{\mathcal{S}_{2}}\left(G^{\prime}\right) \leq 3$, so by Lemma $1 \chi_{u g}^{\mathcal{S}_{2}}(G) \leq 3$.

\section{The Unilateral $\mathcal{S}_{d}$-Colouring Game on Graphs from $\mathcal{H}_{4}$}

In this section we prove the following theorem

Theorem 6 Every graph $G \in \mathcal{H}_{4}$ satisfies $\chi_{u g}^{\mathcal{S}_{d}}(G) \leq 6-d$ for $d \in\{2,3\}$. 
Similarly, as in Sect. 6, for any connected graph $G \in \mathcal{H}_{4}$ such that each block of $G$ is $K_{4}$ we construct a game closed family $\alpha_{d}(G)$.

Let $d \in\{2,3\}$ and $G$ be a connected graph such that each block of $G$ is $K_{4}$.

Definition 4 (Family $\alpha_{d}(G)$ ) The family $\alpha_{d}(G)$ contains the uncoloured graph $G$ and partially $(6-d)$-coloured graphs from $\mathcal{G}^{(6-d)}$ with the monochromatic center $S$ such that every uncoloured vertex $u$ has one of the following properties

1. The vertex $u$ has exactly one coloured stem and there are at most three similar front-neighbours of $B_{b}^{\prime}(u)$, i.e.,

$$
\mid \bigcup_{x \in C \cap B_{b}^{\prime}(u)}\left\{y \mid y \in N_{f}(x) \text { and } c(y)=c(x)\right\} \mid \leq 3 \text {, }
$$

and if $B_{b}^{\prime}(u)$ has three similar front-neighbours, then $u$ is the only uncoloured vertex of $B_{b}^{\prime}(u)$.

2. The vertex $u$ has exactly two coloured stems and

(a) every uncoloured vertex of $B_{b}^{\prime}(u) \backslash\{u\}$ has one coloured stem and every coloured vertex of $B_{b}^{\prime}(u)$ has no similar front-neighbour,

$\left(b_{1}\right)$ in $\mathcal{B}_{f}(u) \backslash\{u\}$ there is an uncoloured vertex with two coloured stems, and every coloured vertex of $\mathcal{B}_{f}(u)$ has no similar front-neighbour, and there are not any two vertices coloured with the same colour, or

$\left(\mathrm{b}_{2}\right)$ every uncoloured vertex of $\mathcal{B}_{f}(u) \backslash\{u\}$ has exactly one coloured stem and in $\mathcal{B}_{f}(u)$ there are at most two coloured vertices that have a similar frontneighbour and they have at most one similar front-neighbour, and there are at most two vertices with the same colour.

The following example illustrates Definition 4 and the idea of the proof of Lemma 17.

Example 5 To prove that the family $\alpha_{d}(G)$ is $\left(\mathcal{S}_{d}, 6-d\right)$-game closed we must prove that for each $\left(G, c_{6-d}\right) \in \alpha_{d}(G)$, Alice can colour a vertex of $\left(G, c_{6-d}\right)$ in such a way that after her move each uncoloured vertex of $\left(G, c_{6-d}\right)$ has either the property (1) or (2) of Definition 4 and if Bob colours a vertex of $\left(G, c_{6-d}\right)$ and after his move there are uncoloured vertices, then there is a vertex that can be coloured by Alice such that the resulting graph belongs to $\alpha_{d}(G)$. Let $G$ be the uncoloured graph isomorphic to the graph in Fig. 5. Observe that the partially 3-coloured graph $\left(G, c_{3}\right)$ (see Fig. 5) belongs to $\alpha_{3}(G)$ : let us choose the vertices $\left\{s_{1}, s_{2}, s_{3}, s_{4}\right\}$ to be the monochromatic center $S$, then the uncoloured vertices on the shortest $\left(s_{3}-x_{7}\right)$-path have the property (2), the remaining uncoloured vertices have the property (1). Alice colours an uncoloured neighbour of any vertex of $S$ with 2 , then the resulting graph is in $\alpha_{3}(G)$. Now, we consider some possible moves of Bob. Suppose that Bob colours the vertex $b_{1}$ with 2 . Thus, he destroys the property (1) of the vertex $a_{1}$, since now there are four similar front-neighbours of $B_{b}^{\prime}\left(a_{1}\right)$. Then, Alice colours the vertex $a_{1}$ with 1 . Suppose that Bob colours $b_{2}$, so he destroys the property (1) of the vertex $a_{2}$ and the property (2) of $a_{3}$ (in $B_{b}^{\prime}\left(a_{3}\right)$ there is a vertex with two coloured stems). Alice colours $a_{2}$ with an arbitrary colour or $a_{3}$ with the colour distinct from 3. Suppose that Bob colours 


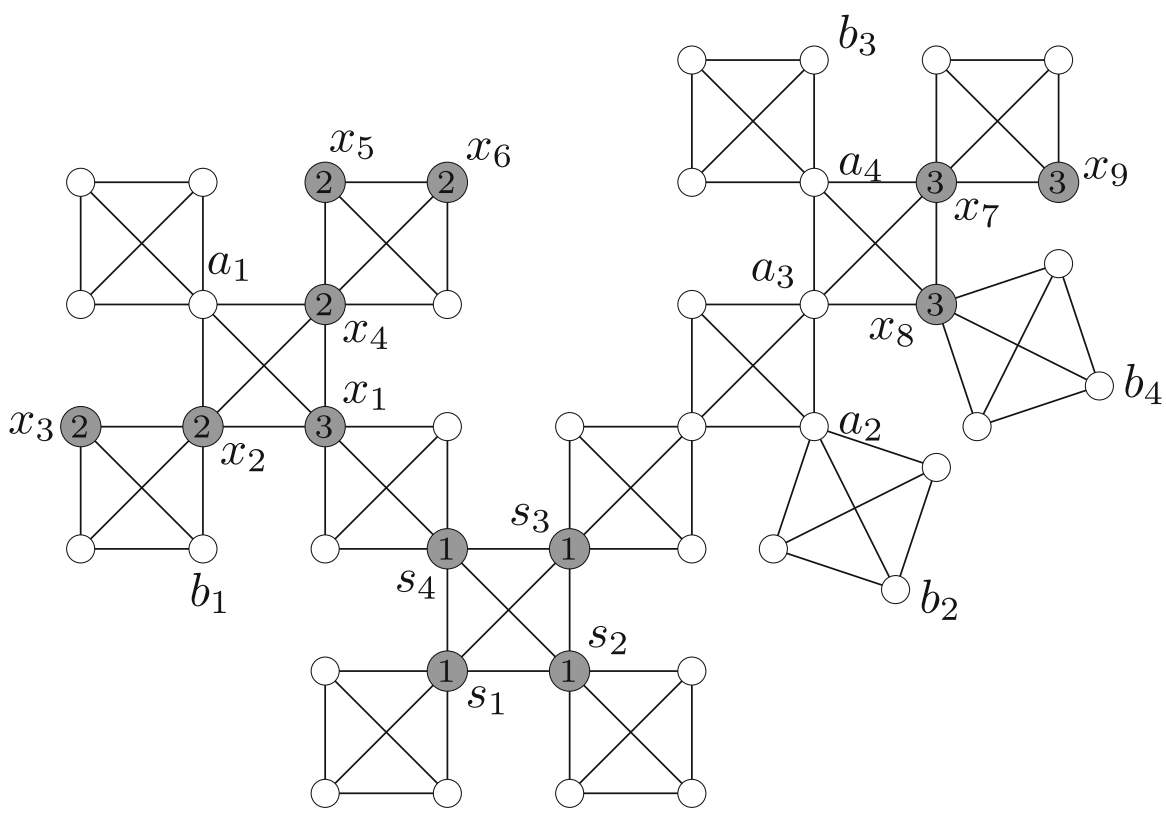

Fig. 5 The graph $G$ in Example 5

the vertex $b_{3}$. Thus, he destroys the property (1) of the vertex $a_{4}$ and the property (2) of the vertex $a_{3}$ (in $\mathcal{B}_{f}\left(a_{3}\right)$ there is the uncoloured vertex $a_{4}$ with two coloured stems and the coloured vertex $x_{7}$ with a similar front-neighbour). Observe that the vertex $a_{4}$ has not the property (2), since in $B_{b}^{\prime}\left(a_{4}\right)$ there is the vertex $x_{7}$ with a similar front-neighbour. Therefore, Alice must colour $a_{4}$. If Bob colours $a_{4}$ with 3 , then he destroys the property (2) of $a_{3}$. Then Alice colours $a_{3}$ with the colour distinct from 3 .

The next remark concerns Definition 4.

Remark 2 Let $B_{f}(u)$ be a block of $\mathcal{B}_{f}(u)$ belonging to the coloured front-stem of $u$. If the vertex $x \in B_{f}(u) \backslash\{u\}$ has two coloured front-stems, then it has the property (2) and $B_{f}(u)$ is the back-block of $x$. Thus, by Condition 2(a) each coloured vertex of $B_{f}(u)$ has no similar front neighbour. Hence, the condition $\left(\mathrm{b}_{1}\right)$ can be equivalently replaced by the following one

$\left(\mathrm{b}_{1}^{\prime}\right)$ in $\mathcal{B}_{f}(u) \backslash\{u\}$ there is a vertex with two coloured stems and there are not any two vertices with the same colour.

Similarly to Lemmas 12 and 13 we can prove the following

Lemma 15 Let $G \in \alpha_{d}(G)$. Then for any uncoloured vertex there is at most one front-stem with coloured vertices.

Lemma 16 Let $G \in \alpha_{d}(G)$. Then in every block there are at most two uncoloured vertices with the property (2). 
Lemma 17 Let $d \in\{2,3\}$. Then the family $\alpha_{d}(G)$ is $\left(\mathcal{S}_{d}, 6-d\right)$-game closed.

Proof Let $G \in \alpha_{d}(G)$. We show that Conditions (i) and (ii) of Definition 1 hold. Note that if $d=2$, then Alice and Bob use 4 colours, if $d=3$, then they use three colours. Assume that Alice starts. If all vertices of $G$ are uncoloured, then Alice colours an arbitrary vertex. Thus, after her move this vertex is the monochromatic center $S$ and every uncoloured vertex of $G$ with $S$ has the property (1) or (2). Suppose that $G$ has at least one coloured vertex. Thus, each vertex of $G$ with the monochromatic center $S$ satisfies either the property (1) or (2). Alice colours a vertex such that every vertex of $G$ with the same monochromatic center $S$ still has either the property (1) or (2). Let $B$ be a block with at least one uncoloured vertex that has a coloured block-cut-vertex $w$ or has no block-cut-vertex. Let $c_{s}=c(w)$ or $c_{s}=c(S)$, whenever a block-cut-vertex of $B$ does not exist. First, suppose that in $B$ there is a vertex $u$ with the property (2). Let $B_{f}(u)$ be a block of $\mathcal{B}_{f}(u)$ belonging to the coloured front-stem. If in $B_{f}(u)$ there is a vertex with two similar neighbours, then it has one similar neighbour in $B_{f}(u)$. If in $B_{f}(u)$ there are two vertices coloured with one colour, let us denote this colour by $c_{0}$. Alice colours $u$ with an admissible colour such that $c(u)$ is distinct from $c_{0}$ and $c_{s}$. After Alice's move all vertices that had had the property (1) before Alice's move, still have the property (1) after her move and all vertices that had had the property (2) still have the property (2). Thus, $G \in \alpha_{d}(G)$. If in $B$ there is no uncoloured vertex with the property (2), then Alice colours the vertex $u$ with the property (1). Alice colours $u$ with an admissible colour distinct from $c_{s}$. Note that Alice can always find such a colour. Indeed, since $B_{b}^{\prime}(u)\left(B=B_{b}(u)\right)$ has at most three similar front-neighbours, it follows that if in $B_{b}^{\prime}(u)$ there are two vertices with more than $d-1$ similar neighbours, then they are coloured with the same colour or one of them is coloured with $c_{s}$, so Alice can find an admissible colour distinct from $c_{s}$. Now, we consider the case when Bob starts. If after his move $G \in \alpha_{d}(G)$, then obviously Alice can colour a vertex in such a way that also after her move $G \in \alpha_{d}(G)$. Thus, assume that after Bob's move $G \notin \alpha_{d}(G)$, we know that before Bob's move every vertex of $G$ with $S$ had had either the property (1) or (2).

Case 1 Bob has coloured the vertex $b$ in such a way that he has destroyed the property (1) of the vertex $u$.

Thus, Bob has coloured a vertex of an uncoloured stem of $u$ or Bob has coloured a front neighbour of $B_{b}^{\prime}(u)$ in such a way that $B_{b}^{\prime}(u)$ has three similar front-neighbours and in $B_{b}^{\prime}(u) \backslash\{u\}$ there is an uncoloured vertex or $B_{b}^{\prime}(u)$ has four similar frontneighbours.

Case 1.1 The vertex $u$ has two coloured stems.

Note that if $u$ has the property (2), then $G \in \alpha_{d}(G)$. So, we may assume that $u$ does not have the property (2) and hence in $B_{b}^{\prime}(u) \backslash\{u\}$ there is an uncoloured vertex with two coloured stems or a coloured vertex with a similar front-neighbour.

First, suppose that $B_{b}(u)$ has the coloured block-cut-vertex $w$ or $B_{b}(u)$ has no block-cut-vertex. Let $c_{s}=c(w)$ or $c_{s}=c(S)$ if the block-cut-vertex of $B_{b}(u)$ does not exist. Suppose that in $B_{b}(u) \backslash\{u\}$ there is an uncoloured vertex $x$ with two coloured 
stems. Note that $x$ has neither the property (1) nor the property (2). Then Alice colours $u$ with a colour such that $c(u)$ is distinct from $c(b)$ and $c_{s}$. After her move every vertex of $B_{b}^{\prime}(u)$ has no similar front-neighbour and hence $x$ has the property (2), so $G \in \alpha_{d}(G)$. Otherwise, if in $B_{b}(u) \backslash\{u\}$ there is no uncoloured vertex with two coloured stems, Alice colours $u$ with an admissible colour distinct from $c_{s}$. We show that there is an admissible colour for $u$ that is distinct from $c_{s}$. Since $B_{b}^{\prime}(u)$ has at most three similar front neighbours, it follows that if in $B_{b}^{\prime}(u)$ there are two vertices with more than $d-1$ similar front neighbours, then they are coloured with the same colour. Since players use at least three colours, there exists an admissible colour for $u$ distinct from $c_{s}$. After Alice's move each uncoloured vertex of $B_{b}^{\prime}(u)$ has the property (1).

Now, assume that $B_{b}(u)$ has the uncoloured block-cut-vertex $w$. Let $x, y \in B_{b}(u) \backslash$ $\{u, w\}$. If $x$ and $y$ are coloured, then they have at most one similar front-neighbour. If $c(x)=c(y)=c$, then Alice colours $u$ in such a way that $c(u) \neq c(b)$ and $c(u) \neq c$. If $c(x) \neq c(y)$, then Alice colours $u$ with $c(x)$ or $c(y)$. Otherwise, i.e., if $x, y$ are uncoloured, then Alice colours $u$ with an admissible colour such that $c(u) \neq c(b)$ and $c(u) \neq c\left(B_{b}(u)\right)$. After such a move $w$ has the property (2) and if in $B_{b}(u)$ was an uncoloured vertex that had two coloured stems, then it has the property (2).

Case 1.2 $B_{b}^{\prime}(u)$ has three similar front-neighbours and in $B_{b}^{\prime}(u) \backslash\{u\}$ there is an uncoloured vertex or $B_{b}^{\prime}(u)$ has four similar front-neighbours.

Since $B_{b}^{\prime}(u)$ has at least three similar front-neighbours, in $B_{b}(u)$ there is no uncoloured vertex with two coloured stems. Therefore, $B_{b}(u)$ has the coloured block-cut-vertex $w$ or $B_{b}(u)$ has no block-cut-vertex. Let $c_{s}=c\left(B_{b}(u) \backslash B_{b}^{\prime}(u)\right)$. Alice colours $u$ with an admissible colour such that $c(u)$ is distinct from $c_{s}$. It is easy to see that such a colour exists.

Case 2 Bob has coloured the vertex $b$ in such a way that he has destroyed the property (2) of the vertex $u$.

Subcase 2.1 The vertex $u$ has three coloured stems.

Thus, Bob has coloured a vertex of an uncoloured front-stem of $u$. If $b$ is adjacent to $u$, then all uncoloured vertices of the stem of $u$ containing $b$ have the property (1). If $b$ is not adjacent to $u$, then vertices that are separators of $b$ and $u$ have the property (2) (before Bob's move they had had the property 1) all the other vertices of this stem have the property (1). Let $B_{f}(u)$ be a block of $\mathcal{B}_{f}(u)$ belonging to a coloured front-stem that does not contain $b$.

Assume that $B_{b}(u)$ has the coloured block-cut-vertex $w$ or $B_{b}(u)$ has no block-cutvertex. Let $c_{s}=c\left(B_{b}(u) \backslash B_{b}^{\prime}(u)\right)$. Then Alice colours $u$ with an admissible colour $c$ such that in $B_{f}(u)$ there are not any two vertices coloured with $c$ and $c \neq c_{s}$. After such a move $B_{b}^{\prime}(u)$ has at most two similar front-neighbours and hence if in $B_{b}(u)$ there is an uncoloured vertex, then it has the property (1). Therefore, $G \in \alpha_{d}(G)$.

Suppose that the block-cut-vertex $w$ of $B_{b}(u)$ is uncoloured. Alice colours $u$ with colour $c$ such that in $\mathcal{B}_{f}(u)$ there is at most one vertex coloured with $c$. Since every coloured vertex of $B_{f}(u)$ has at most one similar front neighbour and in $B_{b}(u)$ there 
are not any two vertices coloured with the same colour, the colour $c$ is admissible for $u$ and after Alice's move $G \in \alpha_{d}(G)$.

Subcase 2.2 In $B_{b}^{\prime}(u)$ there is a coloured vertex with a similar front-neighbour.

Thus, Bob has coloured the front-neighbour $b$ of the coloured vertex $x \in B_{b}^{\prime}(u)$. Let $B_{f}(u)$ be the block of $\mathcal{B}_{f}(u)$ belonging to the coloured front-stem.

Assume that the block-cut-vertex $w$ of $B_{b}(u)$ is coloured or $B_{b}(u)$ has no blockcut-vertex. Let $c_{s}=c\left(B_{b}(u) \backslash B_{b}^{\prime}(u)\right)$. Alice colours $u$ with an admissible colour distinct from $c_{s}$. From the fact that in $B_{f}(u)$ there are at most two vertices coloured with the same colour, it follows that if in $B_{b}^{\prime}(u)$ there is an uncoloured vertex, then it has the property (1). Hence, $G \in \alpha_{d}(G)$.

Suppose that the block-cut-vertex $w$ of $B_{b}(u)$ is uncoloured. Then Alice colours $u$ with colour $c$ such that in $B_{f}(u)$ there is at most one vertex coloured with $c$. Since every vertex of $B_{f}(u) \cup B_{b}^{\prime}(u)$ has at most one similar front neighbour and in $B_{b}^{\prime}(u)$ there are not any two vertices coloured with the same colour, colour $c$ is admissible for $u$ an after such a move $w$ has the property (2).

Subcase 2.3 In $B_{b}^{\prime}(u) \backslash\{u\}$ there is the uncoloured vertex $x$ with two coloured stems.

Thus, Bob has coloured a vertex belonging to a front-stem of the uncoloured vertex $x$. The vertex $x$ had had the property (1) before the Bob's move. Therefore, such a move was considered in Case 1.1.

Subcase $2.4 \operatorname{In} \mathcal{B}_{f}(u)$ there is a vertex with two similar front-neighbours.

Let $B_{f}(u)$ be the block of $\mathcal{B}_{f}(u)$ that is in the coloured front-stem. Thus, Bob has coloured the front-neighbour $b$ of the coloured vertex $x$ of $B_{f}(u)$. Note, that all uncoloured vertices of $B_{f}(u) \backslash\{u\}$ have the property (1). Assume that the block-cut-vertex $w$ of $B_{b}(u)$ is coloured or $B_{b}(u)$ has no block-cut-vertex. Let $c_{s}=c\left(B_{b}(u) \backslash B_{b}^{\prime}(u)\right)$. Alice colours $u$ with an admissible colour such that $c(u)$ is distinct from $c_{s}$. Suppose that the block-cut-vertex $w$ of $B_{b}(u)$ is uncoloured. If in $B_{f}(u)$ there are two vertices with one colour, then let us denote this colour by $c_{0}$. Therefore, Alice colours $u$ with a colour distinct from $c(b)$ and $c_{0}$. After her move in $B_{b}^{\prime}(u)$ only the vertex $u$ may have a similar front-neighbour and in $B_{b}^{\prime}(u) \backslash\{u\}$ there are not any two vertices coloured with the same colour and hence $w$ has the property (2). Thus, $G \in \alpha_{d}(G)$.

Subcase 2.5 In $B_{f}(u)$, there are three vertices coloured with one colour or there are two vertices $x, y$ coloured with one colour and the uncoloured vertex $z \in B_{f}(u) \backslash$ $\{u, x, y\}$ that has the property $(2)\left(B_{f}(u)\right.$ is the block of $\mathcal{B}_{f}(u)$ belonging to the coloured front-stem).

If the block-cut-vertex $w$ of $B_{b}(u)$ is coloured or $B_{b}(u)$ has no block-cut-vertex, then Alice colours $u$ with an admissible colour that is distinct from $c_{s}=c\left(B_{b}(u) \backslash B^{\prime}(u)\right)$ and $c\left(B_{f}(u)\right)$. If the block-cut-vertex $w$ of $B_{b}(u)$ is uncoloured, then Alice colours $u$ with the colour $c$ such that $c \neq c\left(B_{f}(u)\right)$.

Proof of Theorem 6 Let $G \in \mathcal{S}_{4}$. Let $G^{\prime}$ be the connected graph such that each block of $G^{\prime}$ is $K_{4}$ and $G \subseteq G^{\prime}$. Lemma 17 implies that there exists the $\left(\mathcal{S}_{d}, 6-d\right)$-game 
closed family containing $G^{\prime}$ for $d \in\{2,3\}$, so Alice can use the $\left(G^{\prime} ; \mathcal{S}_{d}, 6-d\right)$ strategy to win the unilateral $\mathcal{S}_{d}$-colouring game on $G^{\prime}$ with $6-d$ colours. Thus, by Lemma 1 we have $\chi_{u g}^{\mathcal{S}_{d}}(G) \leq 6-d$.

\section{Conclusion}

In [15], the $r$-colouring game and the $(r, 1)$-relaxed colouring game on graphs from $\mathcal{H}_{k}$ were considered and the following theorem was proved.

Theorem 7 ([15]) For $k \geq 2, \chi_{g}\left(\mathcal{H}_{k}\right) \leq k+2$ and $\chi_{g}^{(1)}\left(\mathcal{H}_{k}\right) \leq k+1$.

Moreover, it was shown that the first result can be improved for all $k \geq 6$, i.e., $\chi_{g}\left(\mathcal{H}_{k}\right) \leq k+1(k \geq 6)$.

Lemma 7 and Theorems 5, 7 imply the following

Corollary $2 \chi_{g}^{(d)}\left(\mathcal{H}_{3}\right) \leq 5-d$ for $0 \leq d \leq 2$.

From Lemma 7 and Theorems 6, 7 we immediately have

Corollary $3 \chi_{g}^{(d)}\left(\mathcal{H}_{4}\right) \leq 6-d$ for $0 \leq d \leq 3$.

We combine Corollaries 2, 3 in the following formula

Corollary 4 For $k \in\{3,4\}$ and $0 \leq d \leq k-1$ it holds that $\chi_{g}^{(d)}\left(\mathcal{H}_{k}\right) \leq k+2-d$.

For $d=k$ the problems remain still open. For $d=k$ we have $k+2-d=2$, thus we can reformulate the problem in the following way: find the smallest $d$ for which Alice has a winning strategy for the $d$-relaxed colouring game on graphs from $\mathcal{H}_{3}$ (or $\mathcal{H}_{4}$ ) with two colours. Note that using the properties of the unilateral $S_{d}$-colouring game (Lemma 9) from Theorems 5 and 6 we have

Corollary 5 If $d \geq 2$, then $\chi_{g}^{(d)}\left(\mathcal{H}_{3}\right) \leq 3$.

Corollary 6 If $d \geq 3$, then $\chi_{g}^{(d)}\left(\mathcal{H}_{4}\right) \leq 3$.

Similarly to the generalized game, we can generalize the unilateral $\mathcal{P}$-colouring game to the unilateral $\left(\mathcal{P}_{1}, \mathcal{P}_{2}, \ldots, \mathcal{P}_{r}\right)$-colouring game. More precisely, let be given an ordered set of additive hereditary properties $\left(\mathcal{P}_{1}, \mathcal{P}_{2}, \ldots, \mathcal{P}_{r}\right)$, a graph $G$ and a set of colours $\{1, \ldots, r\}$. The unilateral $\left(\mathcal{P}_{1}, \mathcal{P}_{2}, \ldots, \mathcal{P}_{r}\right)$-colouring game on $G$ is played by two players, Alice and Bob, who colour vertices of $G$. Alice starts the game. The players play alternatively, but Bob can miss his move. Bob may colour the vertex with an arbitrary colour from $\{1, \ldots, r\}$, while Alice colours the vertex with colour $i$ in such a way that she cannot create a monochromatic minimal forbidden subgraph for the property $\mathcal{P}_{i}$. If after $|V(G)|$ moves all vertices of the graph $G$ are coloured, then Alice wins the game, otherwise Bob wins. Similarly to the $(G ; \mathcal{P}, r)$-strategy we can define the $\left(G ; \mathcal{P}_{1}, \mathcal{P}_{2}, \ldots, \mathcal{P}_{r}\right)$-strategy for playing the unilateral $\left(\mathcal{P}_{1}, \mathcal{P}_{2}, \ldots, \mathcal{P}_{r}\right)$ colouring game. The unilateral $\left(\mathcal{P}_{1}, \mathcal{P}_{2}, \ldots, \mathcal{P}_{r}\right)$-colouring game we can use as a tool to study the $\left(\mathcal{P}_{1}, \mathcal{P}_{2}, \ldots, \mathcal{P}_{r}\right)$-colouring game. 
Acknowledgments We thank the referee for comments that helped improve the manuscript.

Open Access This article is distributed under the terms of the Creative Commons Attribution License which permits any use, distribution, and reproduction in any medium, provided the original author(s) and the source are credited.

\section{References}

1. Andre, S.D.: The game chromatic index of forests of maximum degree $\Delta \geq 5$. Discret. Appl. Math. 154, 1317-1323 (2006)

2. Bodlaender, H.L.: On the complexity of some colouring games. Internat. J. Found. Comput. Sci. 2, 133148 (1991)

3. Borowiecki, M., Sidorowicz, E.: Generalized game colouring of graphs. Discret. Math. 307, 12251231 (2007)

4. Cai, L., Zhu, X.: Game chromatic index of $k$-degenerate graphs. J. Graph Theory 36(3), 144-155 (2001)

5. Chou, C., Wang, W., Zhu, X.: Relaxed game chromatic number of graphs. Discret. Math. 262, 89$98(2003)$

6. Dunn, C.: The relaxed game chromatic index of $k$-degenerated graphs. Discret. Math. 307, 17671775 (2007)

7. Dunn, C., Kierstead, H.A.: A simple competitive graph colouring algorithm (II). J. Combin. Theory Ser. B 90, 93-106 (2004)

8. Dunn, C., Kierstead, H.A.: A simple competitive graph colouring algorithm (III). J. Combin. Theory Ser. B 90, 137-150 (2004)

9. Erdös, P.L., Faigle, U., Hochstättler, W., Kern, W.: Note on the game chromatic index of trees. Theor. Comput. Sci. 313, 371-376 (2004)

10. Faigle, U., Kern, U., Kierstead, H.A., Trotter, W.T.: On the game chromatic number of some classes of graphs. Ars Combin. 35, 143-150 (1993)

11. Guan, D., Zhu, X.: The game chromatic number of outerplanar graphs. J. Graph Theory 30, 67-70 (1999)

12. He, W., Wu, J., Zhu, X.: Relaxed game chromatic number of trees and outerplanar graphs. Discret. Math. 281, 209-219 (2004)

13. Kierstead, H.A.: A simple competitive graph colouring algorithm. J. Combin. Theory Ser. B 78, 57-68 (2000)

14. Kierstead, H.A., Trotter, W.T.: Competitive colorings of oriented graphs. Electron. J. Combin. 8(12) (2001)

15. Sidorowicz, E.: Colouring game and generalized colouring game on graphs with cut-vertices. Discuss. Math. Graph Theory 30, 499-533 (2010)

16. Wu, J., Zhu, X.: Relaxed game chromatic number of outerplanar graphs. Ars Combin. 81, 359-367 (2006)

17. Wu, J., Zhu, X.: The 6-relaxed game chromatic number of outerplanar graphs. Discret. Math. 308, 5974-5980 (2008)

18. Yang, D., Zhu, X.: Activation strategy for asymmetric marking games. Eur. J. Comb. 29, 1123-1132 (2008)

19. Yang, D.: Activation strategy for relaxed asymmetric coloring games. Discret. Math. 309, 33233335 (2009)

20. Zhu, X.: The Game Colouring Number of Planar Graphs. J. Combin. Theory Ser. B 75, 245-258 (1999) 